\title{
Le discage des carottes : estimation des contraintes in situ et optimisation du carottage
}

\section{Core discing : in situ stresses evaluation and coring optimization}

\author{
V. VENET, J.-P. HENRY
}

Laboratoire de Mécanique de Lille - URA CNRS 1441

Département de Mécanique des Matériaux Fragiles EUDIL"

D. FOURMAINTRAUX

Expertise Mécanique des roches Elf Aquitaine *

Rev. Franç. Géotech. $n^{\circ} 61$, pp. $75-84$ (décembre 1992)

\section{Résumé}

Le discage des carottes est un phénomène qui peut être utilisé pour :

- I'estimation des contraintes in situ à partir d'observation de carottes disquées ;

- l'optimisation des paramètres de carottage afin d'obtenir des carottes intactes pour les essais mécaniques en laboratoire.

Dans un premier temps, nous décrirons le logiciel utilisant la méthode des éléments finis (DISCAGELF) développé pour notre étude. Nous présenterons ensuite les résultats des essais effectués sur le triaxial vrai de I'Université de Lille (sans pression de boue), et sur la machine à disquer de I'Institut National de l'Environnement et des Risques Industriels (INERIS) (avec pression de boue simulée dans la saignée) pour valider notre logiciel. Puis, nous résumerons les principaux résultats numériques relatifs à l'influence des para. mètres (propriétés géomécaniques du matériau, densité de boue, poids sur l'outil, rapport des rayons du carottier, type et géométrie de l'outil) sur les contraintes d'initialisation de la rupture. Nous avons principalement mis en évidence l'existence :

- d'un domaine de sécurité vis-à-vis de l'amorce de la rupture ;

- de relations linéaires entre les contraintes radiales d'initialisation de la rupture d'une part, et les propriétés géomécaniques de la roche et la pression de boue d'autre part ; - de trois positions préférentielles de l'amorce de la rupture (le mécanisme engendrant la fracture étant différent) :

- de l'influence du type et de la géométrie du carottier sur l'initialisation de la fracture.

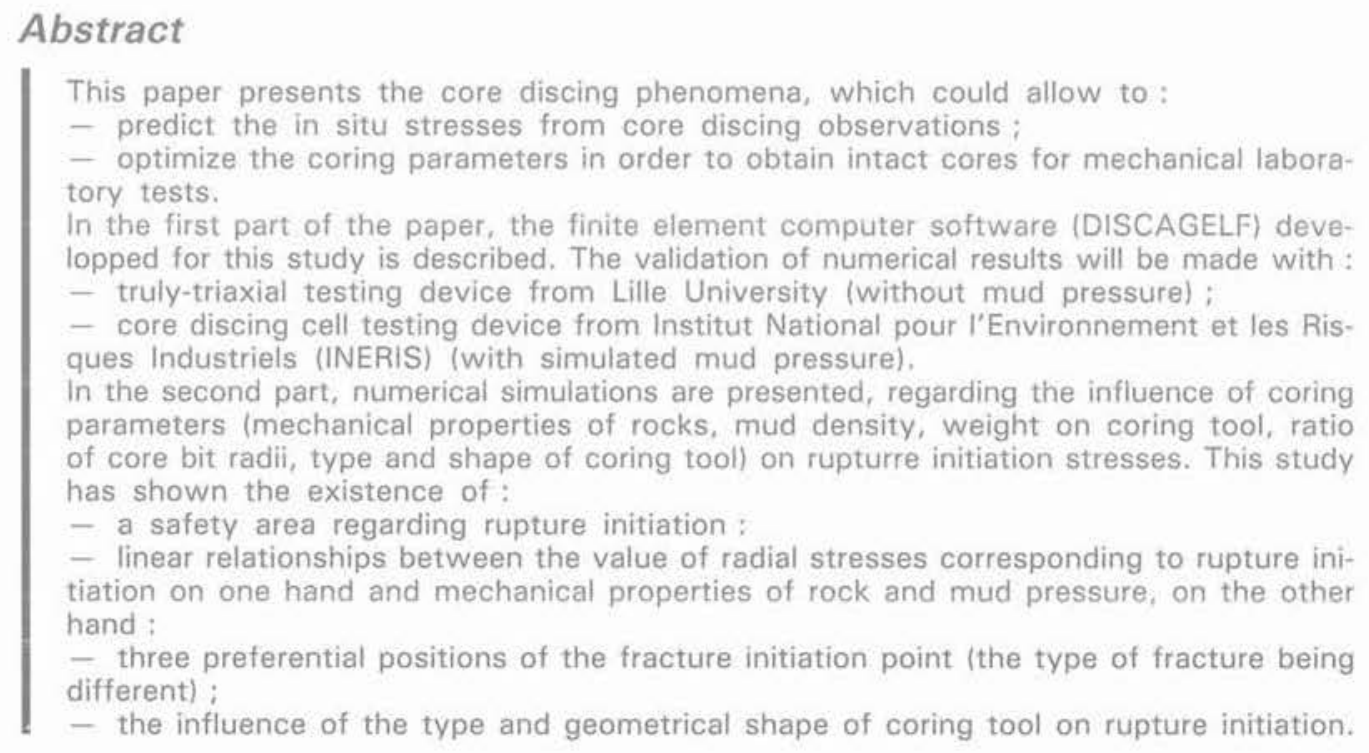

- 59655 Villeneuve-d'Asca Cedex.

* Centre Micauleau, av. Président-Argot, 64001 Pau. 


\section{INTRODUCTION}

Un problème fréquent en mécanique des roches appliquée aux forages pétroliers est l'estimation des contraintes in situ à de grandes profondeurs. Certaines techniques, relativement élaborées, permettent d'obtenir de bonnes indications, sur l'orientation des contraintes horizontales minimum et maximum (ovalisation), sur la valeur de la contrainte horizontale minimum (mini-fracturation hydraulique), sur l'orientation des contraintes principales (analyse de la courbe des déformations différentielles : Differential Strains Curve Analysis (DSCA). Les inconvénients de ces méthodes proviennent de problèmes de faisabilité, de représentativité des résultats obtenus, et principalement des coûts élevés de mise en œuvre.

Dans certains forages pétroliers, on a pu observer le découpage des carottes extraites en tronçons appelés " disques". Ces disques sont " de formes variées, d'épaisseur uniforme avec des surfaces de rupture pratiquement normales à l'axe du forage * (9). Ce phénomène intervient principalement dans des zones fortement contraintes et/ou à grande profondeur. Il apparaît donc lié aux contraintes en place d'où l'idée de l'utiliser comme un indicateur de l'état de contraintes in situ.

La méthodologie retenue pour l'étude du discage comporte deux étapes :

- la première phase consiste à modéliser numériquement lamorce de la rupture en utilisant une approche par critère. En tenant compte de l'ensemble des paramètres du forage, les contraintes d'initialisation de la rupture et les positions des points correspondants seront définies. Une étude expérimentale doit alors être mise en œuvre pour valider les résultats numériques ;

- dans une deuxième phase, à partir des résultats obtenus sur l'armorce de la rupture, il est possible:

- en partant d'une première estimation des contraintes in situ, d'optimiser le carottage de façon à obtenir des carottes intactes pour des mesures pétrophysiques et des essais mécaniques en laboratoire:

- de modéliser la propagation de la fissure en utilisant une approche par la mécanique linéaire de la rupture. On pourrait ainsi obtenir des indications sur la forme des disques et sur les contraintes de discage. A l'inverse, l'observation des carottes disquées interprétée en fonction des paramètres du forage, permettrait d'estimer l'état de contrainte in situ.

Nous n'aborderons, dans cet article, que la première phase de l'étude du discage. Nous définirons ainsi les paramètres ayant une influence sur l'amorce de la rupture, ce qui nous donnera des indications sur la façon d'obtenir des carottes intactes. Après un rappel succinct de l'analyse bibliographique qui explicitera les principaux paramètres de l'étude, nous présenterons le modèle numérique et le logiciel de calcul mis au point: DISCAGELF. La validation du modèle proposé est établie par différents essais en laboratoire. Les résultats essentiels, obtenus lors de l'étude paramétrique de l'initialisation du discage réalisée à l'aide du modèle numérique, permettront de conclure sur les interprétations possibles et les développements futurs.

\section{ANALYSE BIBLIOGRAPHIQUE PRÉSENTATION DES PARAMĖTRES ÉTUDIÉS}

Les principales relations numériques et empiriques relevées dans différentes études antérieures montrent que :

- la contrainte radiale de discage $\left(\sigma_{R}\right)$ varie linéairement en fonction de la contrainte axiale $\left(\sigma_{A}\right)$ : $\sigma_{\mathrm{R}}=\mathrm{k}_{1}+\mathrm{k}_{2} \sigma_{\mathrm{A}}$. Cette équation est celle de la droite dite d'OBERT et STEPHENSON (9) ;

- la pente $k_{2}$ de cette droite varie linéairement en fonction de la résistance à la traction du matériau $\left(R_{4}\right): k_{2}=0,25+0,6510^{-2} R_{t}\left(R_{t}\right.$ étant exprimé en $\mathrm{MPa}$ (11) ;

- lordonnée à lorigine $k_{1}$ de cette droite varie linéairement en fonction de la résistance à la compression $\left(\mathrm{R}_{c}\right)$ ou au cisaillement $\left(\tau_{0}\right)$ de la roche (3) (8) (9) (14).

L'influence du diamètre du carottier sur l'épaisseur des disques a été soulignée par ISAEV (6), ZHU LI et WANG (16). La relation entre le rapport épaisseur des disques sur diamètre du carottier et la concentration de contrainte en fond de saignée a été mise en évidence par STACEY (12), SUGAWARA et al. (13).

Toutes ces données sont issues d'observations d'essais en laboratoire, de simulations numériques et ne tiennent pas compte de la pression de boue dans le forage. On remarque que les paramètres influençant le discage et évoqués par les différents auteurs, se regroupent en deux classes. Les uns sont " passifs » tels que caractéristiques géomécaniques de la roche, longueur de la carotte, et les autres « actifs » tels que densité de boue, poids sur l'outil, rapport des rayons du carottier, forme et géométrie de loutil. Ces différents paramètres sont récapitulés sur la figure 1.

En ce qui concerne l'observation des carottes disquées, signalons les travaux de MIGUEZ (7) qui a élaboré une méthodologie de description des disques (cinq familles de disques ont pu être ainsi répertoriées selon leur forme: assiette, gouttière, selle de cheval, assiette inversée, gouttière inversée).

\section{PRÉSENTATION DU MODĖLE NUMÉRIQUE ET DU LOGICIEL. DISCAGELF}

\subsection{Description générale de DISCAGELF}

L'idée de base est de développer un logiciel, facile d'accès, aisé à manipuler et qui puisse être implanté sur des micro-ordinateurs afin d'être utilisé sur "sites » par les ingénieurs pétroliers. Le principal objectif étant d'effectuer une étude paramétrique complète de l'incidence de certains facteurs sur l'initilisation de la rup- 


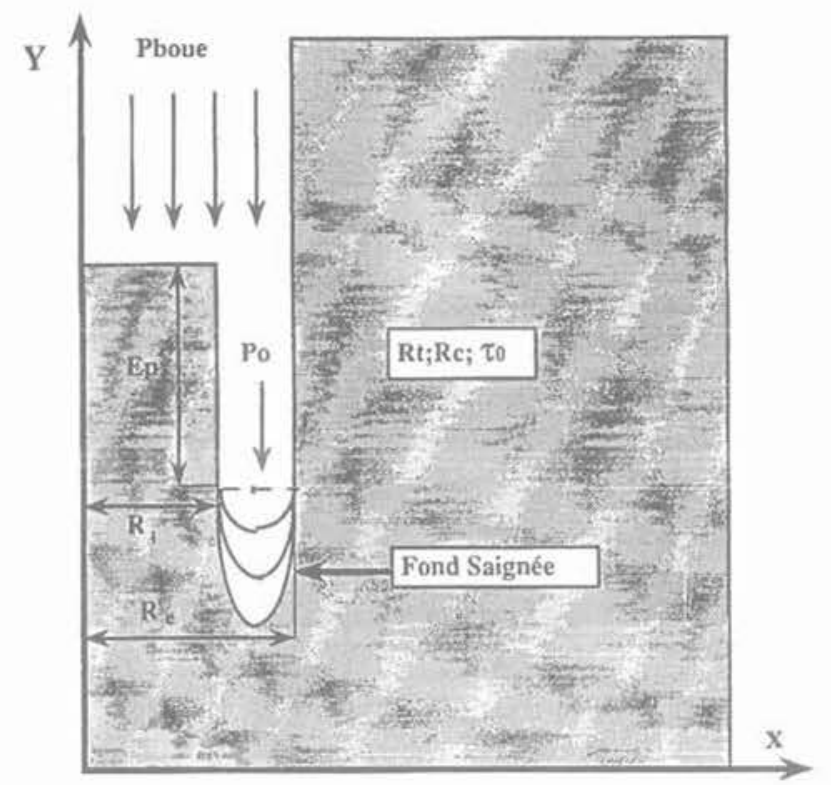

Fig. 1. - Présentation des paramètres testés.

Fig. 1, - Presentation of the testing parameters.

ture, la loi de comportement du matériau sera la plus simple possible: la roche est supposée élastique linéaire homogène et isotrope. La méthode de calcul est celle des éléments finis en symétrie de révolution, ce qui impose deux autres hypothèses simplificatrices: la contrainte dans l'axe du forage est supposée principale, et dans le plan perpendiculaire, les contraintes radiales sont supposées identiques, ce qui est très simplifié par rapport à l'état de contraintes naturelles plus courant où les trois contraintes principales sont différentes. Les éléments de la discrétisation sont des quadrilatères à huit nouds et quatre points de Gauss. Notre logiciel se compose :

- d'un mailleur automatique qui permet de discrétiser totalement le domaine sans intervention extérieure. Le nombre d'entrées est limité à un numéro (IMAIL) correspondant à un type et à une géométrie du carottier :

- d'un dispositif de loupe permettant de focaliser le domaine d'étude sur la zone à haute probabilité de rupture :

- d'une possibilité de sortie graphique des résultats sous formes de courbes d'isovaleurs (courbes isocontraintes-isocritères...) par l'intermédiaire d'un postprocesseur ISOVAL. La figure 2 représente de façon succincte l'organigramme général de DISCAGELF.

Lors de la phase de calcul, quatre modes de chargement sont testés séparément : contrainte axiale seule, contrainte radiale seule, pression de boue seule et poids sur l'outil seul. Les contraintes ainsi obtenues sont stockées dans des fichiers qui seront utilisés lors du calcul de la rupture. Cette méthode pésente lavantage, pour une géométrie du domaine fixée, de faire varier un nombre important de données en superposant différents états de contrainte, sans repasser par l'ensemble du programme de calcul.

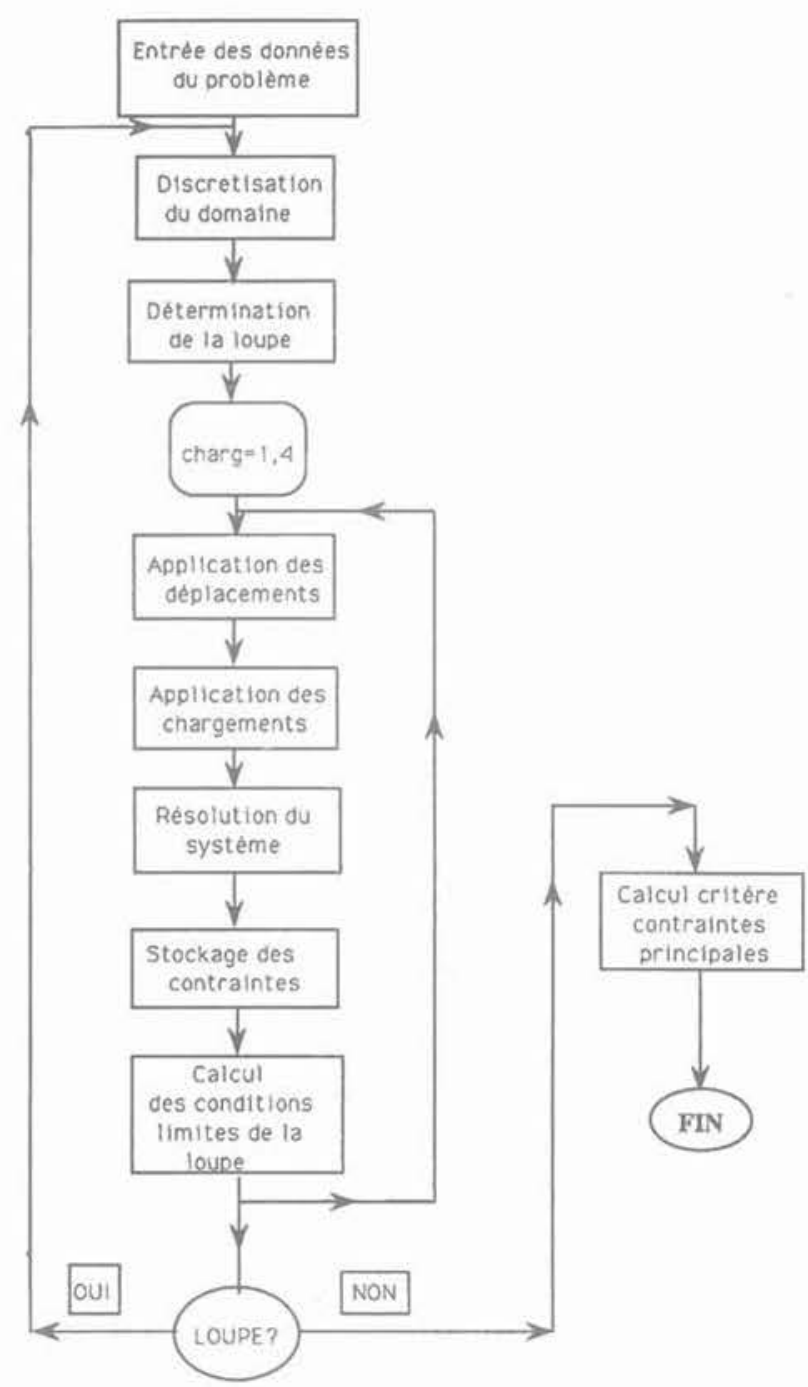

Fig. 2. - Organigramme du logiciel DISCAGELF. Fig, 2 - DISCAGELF software organigram.

\subsection{Description du critère de rupture}

Le critère parabolique de TORRE-LEON, d'écriture simple, a été retenu pour notre étude. Il présente également l'avantage sur un critère de Mohr-Coulomb de ne pas trop surestimer les contraintes principales de rupture dans les zones de forte compression, mais conserve le désavantage de ne pas faire intervenir la contrainte intermédiaire. Il s'écrit :

$$
6 \tau^{2}-8\left(\mathrm{R}_{\mathrm{t}}+\mathrm{R}_{\mathrm{c}}\right) \sigma-\left(\mathrm{R}_{\mathrm{t}}-\mathrm{R}_{\mathrm{c}}\right)^{2}=0
$$

Soient $\sigma_{10}, \sigma_{2}, \sigma_{3} \circ$ les contraintes principales calculées en chaque point de Gauss pour un état de chargement donné; on définira, en tout point du domaine, la variable $\lambda$, appelée paramètre de retour sur la parabole, telle que le système $\sigma_{1}=\lambda \sigma_{1}$, $\sigma_{2}=\lambda \sigma_{2} \circ, \sigma_{3}=\lambda \sigma_{3}$ implique que l'état $\sigma_{1}, \sigma_{2}, \sigma_{3}$ est un état de rupture (fig. 3 ).

Dans ce cas, l'équation de la parabole de TORRELEON (1) devient:

$$
\lambda^{2}\left(\sigma_{3}^{\circ}-\sigma_{1}^{\circ}\right)^{2}-\lambda\left(R_{t}+R_{c}\right)\left(\sigma_{1}^{\circ}+\sigma_{3}^{\circ}\right)+R_{t} R_{c}=0
$$




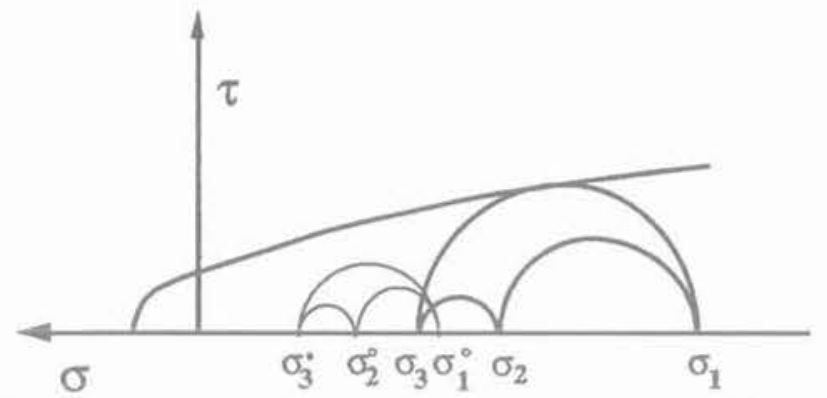

Fig. 3. - Représentation des contraintes principales calculées et de rupture.

Fig. 3. - Representation of principal calculated and rupture stresses.

Au point de calcul considéré, on peut conclure que : - si $\lambda<1$, les contraintes calculées sont trop importantes : la rupture s'est déià produite :

- si $\lambda>1$, les contraintes calculées sont trop faibles: il n'y a pas de rupture :

- si $\lambda=1$, les contraintes calculées vérifient le critère.

L'inconnue du problème est la contrainte radiale $\sigma_{\mathrm{R}}$ (ou le coefficient $\mathrm{K}$ : rapport de la contrainte radiale (horizontale) sur la contrainte axiale (verticale) $\mathrm{K}=$ $\sigma_{\mathrm{R}} / \sigma_{\mathrm{A}}$ ) pour un site (profondeur, nature et densité de la roche, densité de boue) et un carottier (poids sur l'outil, forme du carottier) donnés. En partant de Ihypothèse $\sigma_{\mathrm{A}}=$ poids des terrains et d'une valeur de $\mathrm{K}$ élevée, on effectue des itérations en diminuant ce coefficient jusqu'à ce que l'on obtienne une distribution de contraintes telle que la valeur de $\lambda$ minimale dans tout le domaine soit égale à 1 (à $10^{-4}$ près). La position du point d'initialisation du discage correspond au point où $\lambda$ est égal à 1 .

\subsection{Représentation générale des droites de « discage »}

Lors de chacune de nos simulations, nous avons constaté que (14) (15) :

- la contrainte radiale d'initialisation du discage varie linéairement par intervalles, en fonction de la contrainte axiale :

- pour une profondeur donnée (donc à contrainte axiale fixée), deux valeurs de contraintes radiales $\sigma_{\mathrm{R} 1}$ et $\sigma_{\mathrm{R} 2}$ correspondant à deux valeurs $\mathrm{K}_{1}$ et $\mathrm{K}_{2}$ du coefficient $\mathrm{K}\left(\mathrm{K}_{1}>\mathrm{K}_{2}\right)$, peuvent provoquer l'initialisation du discage.

En reliant entre eux les segments de droite obtenus, on peut définir un domaine à l'intérieur duquel l'amorce de la rupture est impossible et que l'on appellera domaine de non discage (fig. 4).

Les changements de pente et le brusque décrochement sont dus respectivement à des déplacements des points potentiels d'initialisation du discage et à un changement dans le mécanisme qui occasionne la rupture.

Pour $\sigma_{R 2}$, lamorce de rupture apparaît sous la saignée du côté interne, la rupture survenant principalement en extension.

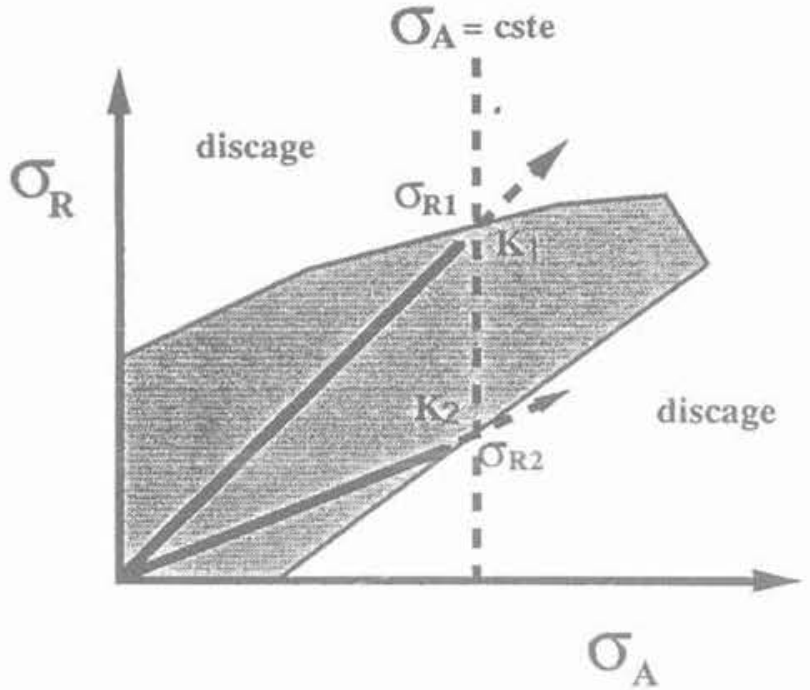

Fig. 4. - Définition du domaine de non-discage dans le plan des contraintes axiale, $\sigma_{A}$, et radiale, $\sigma_{R}$, à la paroi du trou. Pour chaque valeur de la contrainte axiale $\sigma_{A}$, on trouve deux valeurs critiques, $\sigma_{R 1}$ et $\sigma_{R 2}$, de la contrainte radiale $\sigma_{R}$ (correspondant à deux valeurs, $K 1$ et $K 2$, du rapport $K$ entre les contraintes radiale et axiale). Une instabilité (initialisation du discage) apparaît quand la contrainte radiale à la paroi est supérieure à $\sigma_{R}$ ou inférieure à $\sigma_{R 2}$; entre les deux, la stabilité correspond au non-discage.

Fig. 4. - Definiton of no-discing area in the plane of axial, $\sigma_{A}$, and radial, $\sigma_{R}$, stresses at the borehole wall. At each value of axial stress, $\sigma_{A}$. correspond two threshold values of radial stress $\sigma_{R 1}$ and $\sigma_{R 2}$ (with two values of the ratio $K$ between radial and axial stresses), If the radial stress at the borehole wall $\sigma_{R}$ is higher than $\sigma_{R 1}$ or lower than oRe instability occurs at the wall (discing initiation); for values of radial stress between $\sigma_{R 1}$ and $\sigma_{R 2}$. the stability is the no-discing area.

Pour $\sigma_{R 1}$. l'amorce de rupture apparait sous la saignée du côté externe (rupture en cisaillement), ou sous l'axe du carottier (rupture en extension).

Il est donc possible de définir un domaine de sécurité vis-à-vis de l'amorce du discage pour un type de carottier, une pression de boue et un poids sur l'outil donnés. Il semble évident que ce domaine dépende des paramètres précédents (voir paragraphe 5). On peut alors chercher à optimiser la forme du carottier pour que le domaine de non discage soit le plus grand possible, c'est-à-dire pour réduire la probabilité d'endommagement de la carotte.

\section{VALIDATION EXPÉRIMENTALE DU MODĖLE EN ÉLASTICITÉ (15)}

Deux séries d'essais ont été menés en utilisant une chaîne d'émission acoustique, afin de détecter l'initialisation du discage. Les expérimentations ont eu lieu sur un grès des Vosges (Grès de Wissembourg) avec un carottier de laboratoire de diamètre intérieur $30 \mathrm{~mm}$.

\subsection{Essais sous contraintes triaxiales vraies}

Les premières expérimentations (BOUMADHI, 1) ont été réalisées sur l'appareil triaxial vrai (simulateur 
d'ouvrages souterrains du Laboratoire de Mécanique de Lille) sans présence de boue dans la saignée. Les essais ont été réalisés sur des éprouvettes cubiques $\left(50 \times 50 \times 50 \mathrm{~cm}^{3}\right)$ de calcaire d'Anstrude. L'échantillon étant placé dans le triaxial vrai, on carotte une longueur de carotte (Ep) désirée. Les deux contraintes radiales appliquées sont identiques, le coefficient de poussée des terrains $\mathrm{K}$ (rapport de la contrainte radiale $\sigma_{\mathrm{R}}$ sur la contrainte axiale $\sigma_{\mathrm{A}}$ ) est fixé pour chaque essai. Une chaîne d'émission acoustique est employée afin de détecter le début de l'amorce de rupture (correspondant à $\sigma_{\mathrm{R} 1}$ ) grâce à un capteur piézoélectrique situé sur la carotte. On désirait observer les valeurs de $\sigma_{\mathrm{R}}$ correspondant à différentes longueurs de carottes pour différents coefficients K. Le jeu de données d'entrées pour les simulations numériques correspondantes incluait les caractéristiques géomécaniques de la roche testée, ainsi que la géométrie et la forme du carottier utilisé.

Les contraintes $\sigma_{\mathrm{R} 1}$ obtenues numériquement et expérimentalement sont identiques (écart $<5 \%$ ). Nous avons représenté sur la figure 5 le cas où $\mathrm{K}=3$.

Après l'obtention de la contrainte radiale « d'initialisation du discage $* \sigma_{\mathrm{R} 1}$ BOUMADHI (1) a continué ses essais jusqu'à ce que le disque soit totalement décollé ; la contrainte radiale « de discage " $\left(\sigma_{\mathrm{Rd}}\right)$ est alors relevée. On a pu constater que $\sigma_{R d}$ était très supérieure à $\sigma_{\mathrm{R} 1}$, l'écart entre ces valeurs correspond à la formation du disque. Il est donc important de distinguer l'état d'initialisation du discage et l'état où le disque est totalement formé.

\subsection{Validation expérimentale avec pression de boue}

La deuxième série d'essais a été effectuée sur la cel. lule à disquer de MOUDAFI (8) à l'Institut National

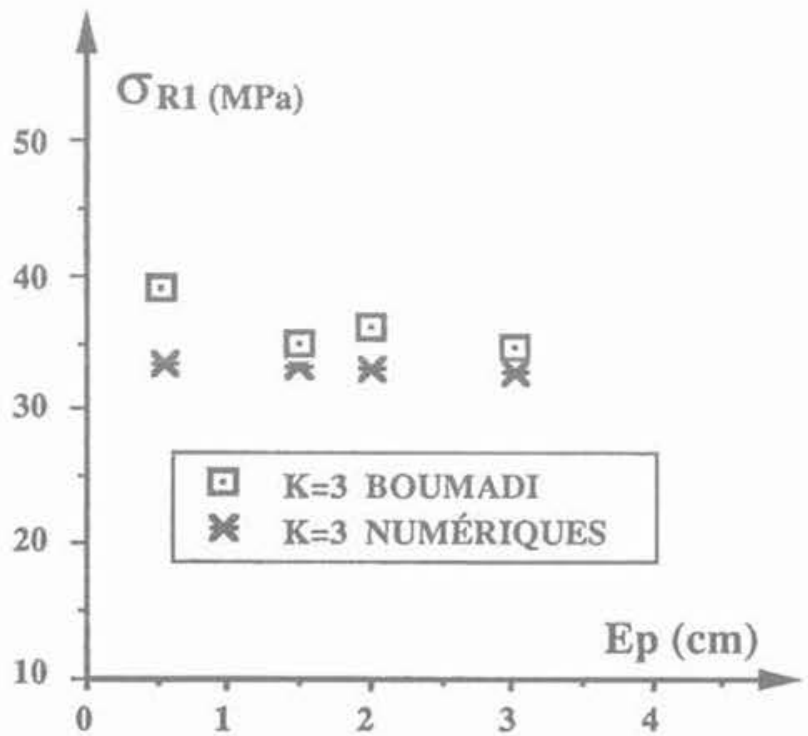

Fig. 5. - Comparaison de résultats expérimentaux d'après BOUMADHI (1) et calculés à l'aide de DISCAGELF. Fig. 5. - Comparison between experimental and calculated values. pour l'Environnement et les Risques Industriels (INERIS). Pour simuler la présence de boue dans la saignée, nous avons employé de la poudre (talc) plutôt qu'un fluide (huile), ce qui nous permet d'éviter la pénétration du liquide dans la roche et de continuer à travailler en contraintes totales.

Le comportement du talc a été étudié au moyen d'essais de compression. Nous avons ainsi pu déterminer le coefficient de transmission de pression $\left(C_{T P}\right)$ en fond de saignée selon le chargement imposé en tête du talc et sa mise en place (préchargement et précompaction). Selon la valeur du $\mathrm{C}_{\mathrm{TP}}$, nous avons pu estimer une "densité de boue équivalente „ à introduire dans la simulation numérique. Le mode opératoire suivi pour chaque expérimentation se décompose en trois grandes phases.

Dans un premier temps, l'échantillon est préparé. Pour cela, après avoir surfacé des éprouvettes de $120 \mathrm{~mm}$ de diamètre et de $240 \mathrm{~mm}$ de longueur de grès des Vosges, on effectue un premier carottage pour extraire une carotte de $5 \mathrm{~cm}$. Puis on recarotte l'échantillon sur $1 \mathrm{~cm}$; cette valeur correspond à la longueur de la carotte prise constante pour tous les essais (afin de garder une même configuration géométrique).

Dans un second temps, on effectue la précompaction du talc qui s'effectue hors de la cellule à disquer selon le processus élaboré en laboratoire (15). Puis le talc est préchargé dans la cellule à disquer en fonction des contraintes axiales $\left(\sigma_{A}\right)$ et sur le talc $\left(\sigma_{\text {talc }}\right)$ qui seront développées lors des essais de discage. Le rapport $\mathrm{K}_{\mathrm{T}}\left(\mathrm{K}_{\mathrm{T}}=\sigma_{\mathrm{A}} / \sigma_{\text {talc }}\right)$ est fixé avant chaque expérimentation et reste constant pendant chaque essai de discage (ce qui nous permet de travailler avec différents $C_{\text {TP }}$ donc de simuler différentes densités de boue). Les contraintes axiales et radiales sont augmentées isotropiquement. Pour chaque palier, on fait crốtre $\sigma_{\text {talc }}$ de façon à garder $\mathrm{K}_{\mathrm{T}}$ constant et ce jusqu'à obtenir la valeur de $\sigma_{\text {talc }}$ qui sera développée pendant les essais.

La troisième phase est l'essai de discage où pour des contraintes axiales et de talc constantes, on augmente la contrainte radiale jusqu'à l'obtention de l'amorce de la rupture $\left(\sigma_{R}\right)$ et de discage $\left(\sigma_{R d}\right)$.

Un contrôle constant de l'état de l'échantillon durant ces deux dernières étapes est effectué grâce à une chaîne d'émission acoustiques reliée à un capteur piézoélectrique placé sur la cellule. On peut ainsi détecter non seulement l'amorce de la rupture et le discage, mais également tout * accident * jaquette défectueuse au montage, amorce de rupture non désirée,...) durant la phase de précompaction et préchargement du talc.

Le jeu de données entré dans DISCAGELF est complété par les valeurs des résistances à la traction et à la compression de la roche (grès des Vosges), et les caractéristiques géométriques du carottier utilisé. Nous présentons dans le tableau 1 les résultats obtenus à partir de quatre " densité de boue " différentes pour une contrainte axiale de l'ordre de $17 \mathrm{MPa}$. Rappelons que l'initialisation du discage a toujours été détectée par émission acoustique. 
Tableau 1. - Variation des valeurs de la contrainte radiale en fonction de la densité de la boue. Valeurs pour différentes densités de boue de la contrainte radiale correspondant à : I'initialisation du disque (ligne $1=$ valeur mesurée expérimentalement - ligne 2 = valeur déterminée par le calcul) et à la formation complète du disque (ligne 3: valeur expérimentale mesurée).

Roche $=$ Grès des Vosges (Grès rouge du Trias).

Table 1 - Variation with mud density of the radial stress values corresponding with discing initiation (measured experimental values = line 7

- calculated values = line 21) and fully achieved discing (measured experimental values $=$ line 3 )

Rock $=$ Vosges Sandstrone (triasic red sandstone).

\begin{tabular}{|c|c|c|c|c|}
\hline $\begin{array}{l}\text { Densité } \\
\text { Contrainte de boue } \\
\text { tadiale }\end{array}$ & 0 & 0,3 & 0,5 & 0,7 \\
\hline $\begin{array}{l}\text { Initialisation de dis- } \\
\text { cage (expérimental) }\end{array}$ & $67 \mathrm{MPa}$ & $71 \mathrm{MPa}$ & $79 \mathrm{MPa}$ & $>73 \mathrm{MPa}$ \\
\hline $\begin{array}{l}\text { Initialisation de la } \\
\text { rupture (numérique) }\end{array}$ & $60 \mathrm{MPa}$ & $65 \mathrm{MPa}$ & $70 \mathrm{MPa}$ & $75 \mathrm{MPa}$ \\
\hline $\begin{array}{l}\text { Initialisation du dis- } \\
\text { que formé expéri- } \\
\text { mentale }\end{array}$ & $78 \mathrm{MPa}$ & & $93 \mathrm{MPa}$ & \\
\hline
\end{tabular}

En raison de problèmes mécaniques, l'essai simulant une densité de boue de 0,7 a dû être interrompu à une contrainte radiale de $73 \mathrm{MPa}$. Aucune amorce de rupture n'ayant été décelée comparativement aux essais précédents, l'initialisation du discage aurait dû se produire pour des contraintes radiales supérieures à cette valeur. On note qu'elle est prévue, numériquement, vers $75 \mathrm{MPa}$.

$\mathrm{Au}$ travers de ces résultats, on constate que ;

- les contraintes radiales d'initialisation de discage expérimentales sont légèrement supérieures à celles prévues par notre logiciel (de l'ordre de $10 \%$ ):

- les contraintes radiales d'initialisation ou de formation du disque augmentent lorsque la "densité de boue " augmente;

- les contraintes radiales correspondant au disque formé sont très supérieures à celles obtenues lors de l'amorce de la rupture.

Nous pouvons conclure qu'il existe une bonne corrélation entre les résultats expérimentaux et numériques, et donc que le modèle utilisé, malgré sa simplicité ou sa rusticité, semble être valable et autorise une étude paramétrique du phénomène.

\section{5. ÉTUDE PARAMÉTRIQUE}

Nous ne présenterons ici que les résultats les plus significatifs des 200 simulations numériques effectuées avec présence de boue dans la saignée (15).

\subsection{Les paramètres passifs}

5.1.1. Caractéristiques géomécaniques du matériau L'augmentation de la résistance de la roche fait crô̂tre le domaine de non discage (fig. 6) et tend à stabiliser la position du point d'initialisation du discage.

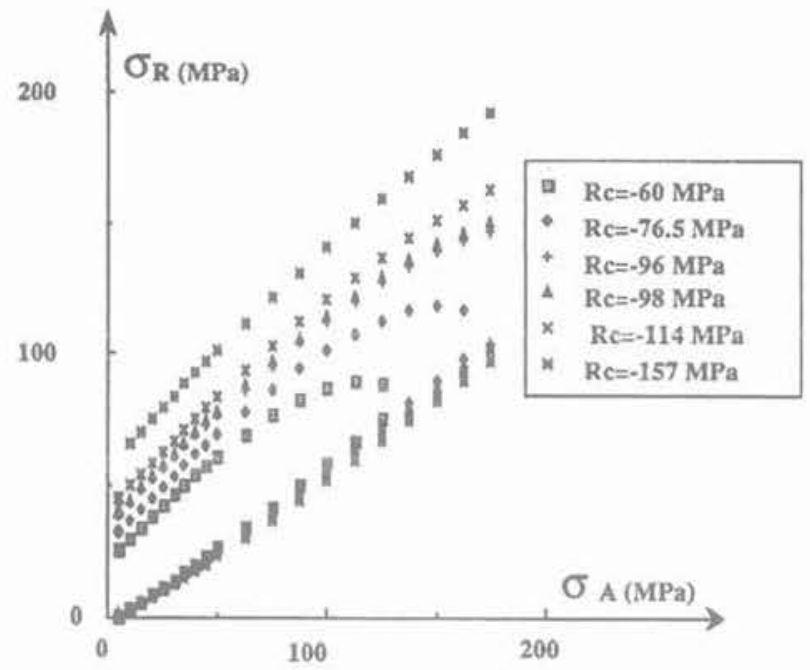

Fig. 6. - Variation du domaine de non discage en fonction de la résistance de la roche $R_{C}$.

Fig. 6. - Variation of no discing domain with rock strength $R_{c}$.

Pour chaque simulation, nous avons constaté que les contraintes radiales d'initialisation de la rupture $\sigma_{\mathrm{R}_{1}}$ et $\sigma_{\mathrm{R} 2}$ (qui sera noté indistinctement $\sigma_{\mathrm{Ri}}$ ) varient linéairement en fonction des résistances à la compression $\left(\mathrm{R}_{c}\right)$ et au cisaillement $\left(\tau_{0}\right)$ du matériau.

On a :

$\begin{cases}\sigma_{\mathrm{Ri}}=\mathrm{A}_{1}+\mathrm{B}_{1} \mathrm{R}_{\mathrm{c}} & \text { (du type MOUDAFI (8)) } \\ \sigma_{\mathrm{Ri}}=\mathrm{A}_{2}+\mathrm{B}_{2} \tau_{0} & \text { (du type OBERT et STE. } \\ & \text { PHENSON (9), DURELLI }\end{cases}$ (3))

Les couples $A_{1}, B_{1}$ et $A_{2}, B_{2}$ sont des fonctions linéaires de la contrainte axiale $\left(\sigma_{\mathrm{A}}\right)$. Pour une géométrie du carottier, une densité de boue et une épaisseur de carotte fixées, on peut écrire ces relations sous la forme:

$$
\sigma_{\mathrm{Ri}}=\mathrm{A}\left(\sigma_{\mathrm{A}}\right)+\mathrm{B}\left(\sigma_{\mathrm{A}}\right) \mathrm{R}_{\mathrm{c}}
$$

En conclusion, en règle générale et comme on pouvait intuitivement l'estimer, plus la roche est résistante, plus le phénomène de discage sera retardé et plus l'extraction de carotte intacte sera favorisée.

\subsubsection{Longueur de la carotte}

(correspondant à l'épaisseur probable des disques)

Deux types de résultats ont été obtenus selon la valeur du rapport des rayons du carottier $\left(\mathrm{R}_{e} / \mathrm{R}_{\mathrm{j}}\right)$ utilisée pour les simulations.

Pour des rapports de rayon du carottier $\left(\mathrm{R}_{e} / \mathrm{R}_{i}\right.$ fixés) inférieurs à 1,7 aucune influence de la longueur de la carotte sur les contraintes radiales d'initialisation du discage n'a été décelée.

Pour des carottiers ayant des rapports de rayon $\left(\mathrm{R}_{e} / \mathrm{R}_{1}\right.$ fixés) surpérieurs à 1,7 ;

- $\sigma_{\mathrm{R} 2}$ reste constante, sans aucune variation quelque soit la longueur de la carotte;

$-\sigma_{\mathrm{R} 1}$ diminue brutalement à partir d'une longueur de carotte supérieure à $2,5 \mathrm{~cm}$. 
Les variations de $\sigma_{\mathrm{R} 1}$ en fonction de la longueur (Ep) sont représentées sur la figure 7 et permettent de distinguer trois * longueurs * de carottes, c'est-à-dire trois familles de disques potentiels :

- d'épaisseur inférieure à $3 \mathrm{~cm}$ : $\sigma_{R 1}$ reste constant :

- d'épaisseur comprise entre $3 \mathrm{~cm}$ et $8 \mathrm{~cm}$ : $\sigma_{\mathrm{R}}$ augmente légèrement ;

- d'épaisseur supérieure à $8 \mathrm{~cm}$ : $\sigma_{\mathrm{R} 1}$ reste constant.

Ce résultat recoupe les observations faites par $\mathrm{ZANON}$ sur de nombreux carottages d'Elf Aquitaine (communication personnelle) faisant apparaître une relation, déjà perçue par ISAEV, entre l'épaisseur des disques et le rapport des rayons du carottier (6).

\subsection{Influence des paramètres actifs}

\subsubsection{Rapport des rayons du carottier $\left(R_{e} / R_{i}\right)$}

Lors de chacune des simulations numériques, l'aug. mentation de ce rapport entraîne, au-delà d'une valeur seuil située vers 1,7 , la diminution très forte de la contrainte radiale d'initialisation du discage $\sigma_{\mathrm{R}}$ (fig. 8) accompagnée d'un brusque changement de la position du point potentiel d'amorce de discage.

Les positions des points d'amorce de la rupture se situent :

- sous la saignée du côté interne, pour la contrainte $\sigma_{\mathrm{R} 2}$;

- sous la saignée du côte externe lorsque $R_{e} / R_{i}$ $<1,7$ ou sous laxe du carottier lorsque $R_{e} / R_{1}$ $>1,7$, pour la contrainte $\sigma_{\mathrm{R} 1}$.

Le rapport des rayons du carottier est donc un paramètre très important car il agit fortement sur les con-

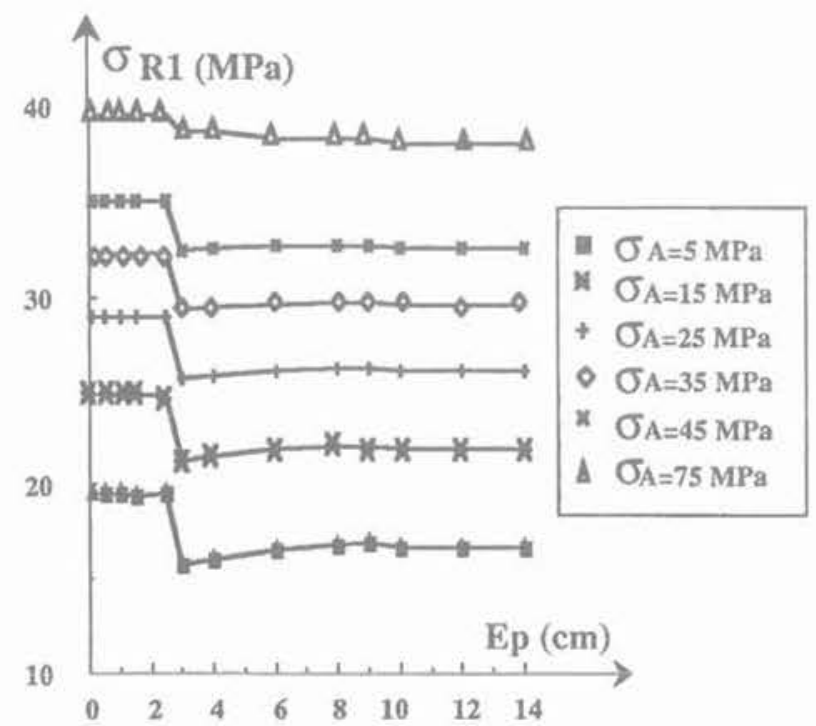

Fig. 7. - Variation de la contrainte radiale critique $\sigma_{R 1}$ et de la longueur de la carotte lc'est-à-dire l'épaisseur du disque) pour différentes valeurs de la contrainte axiale $\sigma_{A}$.

Fig. 7. - Relation between critical radial stress $\sigma_{R 1}$ and core length (i.e. disc thickness) for different values of axial stress $\sigma_{A}$.

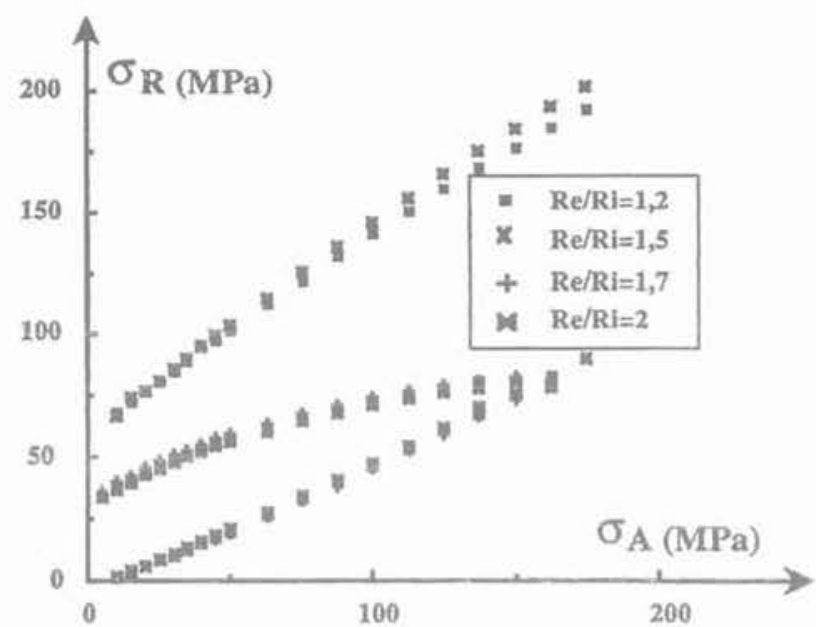

Fig. 8. - Variation du domaine de non discage en fonction du rapport des rayons extérieur $R_{e}$ et intérieur $R_{j}$ du carottier. Fig. 8. - Variation of no-discing domain with the ratio of external $R_{C}$ to internal $R_{j}$ core bit radii.

traintes radiales d'initialisation du discage et sur l'épaisseur des disques potentiels en déplaçant les points d'amorce du discage potentiel.

En résumé, laugmentation du rapport rayon extérieur sur rayon intérieur du carottier favorise lamorce du discage (cette constatation est à rapprocher à celle de DYKE (4) et indiquerait que l'usage de carottiers à surface d'entrée élevée augmente le risque d'endommagement des carottes.

\subsubsection{Densité de la boue}

En règle générale, l'augmentation de la densité de la boue entraîne un accroissement des valeurs des contraintes $\sigma_{\mathrm{R} 1}$ et $\sigma_{\mathrm{R} 2}$, déplaçant ainsi le domaine de non-discage vers des zones de fortes contraintes radiales (fig. 9). Les contraintes $\sigma_{\mathrm{R}}$ varient généralement linéairement en fonction de la densité de lá boue. Les ordonnées à l'origine et les pentes de ces droites varient en fonction de la contrainte axiale. Remarquons que dans certains cas (dépendant du type de carottier, de la nature de la roche), une trop forte densité de boue pourrait provoquer la rupture (ceci rejoint les observations de CHEATHAM (2) et GUÉNOT (5).

On retrouve ici le rôle de la densité de boue vis-àvis des instabilités de la paroi du forage; ici, c'est l'apparition de fissure dans la carotte.

\subsubsection{Poids sur l'outil}

A grande profondeur, et en présence de boue dans la saignée, le poids sur l'outil n'influe pas de manière significative sur les valeurs des contraintes d'amorçage du discage. Pour des profondeurs inférieures à $2000 \mathrm{~m}$, l'augmentation du poids sur loutil agit sur les contraintes $\sigma_{\mathrm{R} 1}$ et $\sigma_{\mathrm{R} 2}$ de la même façon que l'accroissement de la densité de la boue, c'est-à-dire que le domaine de non discage se déplace dans les zones de fortes contraintes radiales; l'amorce de la rupture est alors retardée. 


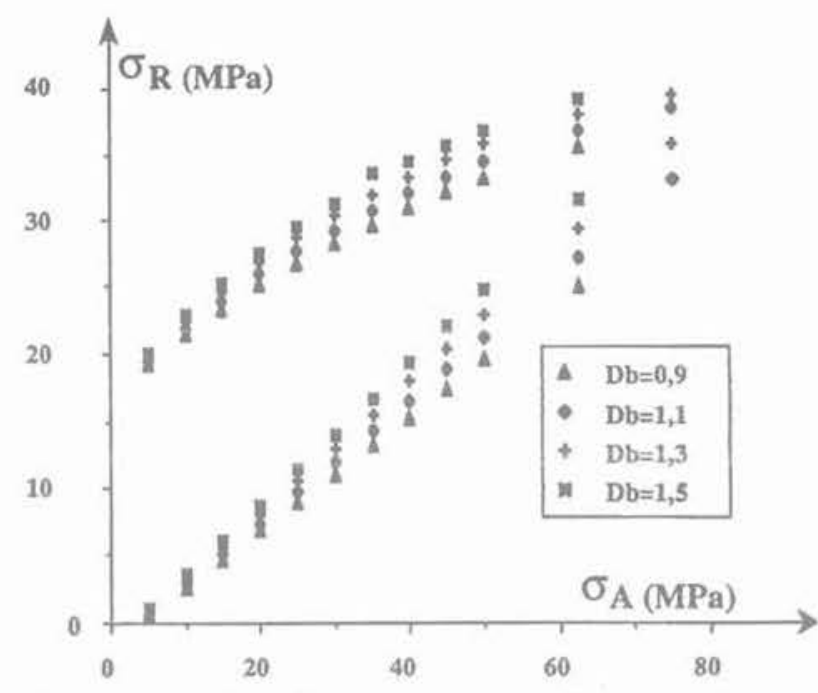

Fig. 9. - Variation du domaine de non discage en fonction de la densité de boue $D_{b}$.

Fig. 9. - Variation of no discing domain with the mud density $D_{b}$.

\subsubsection{Type et géométrie du carottier}

L'influence de tous les facteurs permettant de décrire le fond de la saignée sur les contraintes d'initialisation du discage a été étudiée. Nous nous bornerons, dans cet article, à ne donner que le sens général de variation des valeurs des contraintes $\sigma_{\mathrm{R} 1}$ et $\sigma_{\mathrm{R} 2}$ selon l'excentricité gauche AX1, l'élancement BY et le type de carottier définis sur la figure 10.

Aucune possibilité d'écrire simplement les équations liant $\sigma_{\mathrm{R} 1}$ et $\sigma_{\mathrm{R} 2}$ à ces différents paramètres n'apparaît. Nous avons regroupé dans le tableau 2 les résultats essentiels et tendances générales (quelques exceptions existent).

Nous avons observé qu'un deuxième groupe de paramètres (profondeur du forage, caractéristiques géomécaniques de la roche, densité de la boue) pouvait influer sur le sens de variation des contraintes $\sigma_{\mathrm{R} 1}$ et $\sigma_{\mathrm{R} 2}$. Pour exemple, nous avons représenté sur les figures $11 \mathrm{a}$ et $11 \mathrm{~b}$ le type de graphe obtenu pouvant être utilisé pour des applications au domaine pétrolier.

En superposant ces deux figures (pour une profondeur du puits, une densité de boue et des caractéristiques géomécaniques fixées), on constate que, dans ce cas, le maximum de sécurité vis-à-vis de l'amorce de la rupture est obtenue pour un carottier de type 6 , d'élancement égal à la moitié de l'excentricité gauche $\left(\sigma_{\mathrm{R} 1}\right.$ maximum, $\sigma_{\mathrm{R} 2}$ minimum).

\section{CONCLUSIONS}

Nous avons présenté, dans cet article, une technique d'estimation de l'optimisation du carottage en vue de l'obtention de carottes intactes. Cette technique est tirée de l'étude du phénomène de discage utilisé comme un indicateur des contraintes in situ. Le logiciel DISCAGELF, validé expérimentalement et incluant le maximum de paramètres de forage, est parfaitement adaptable aux besoins des ingénieurs

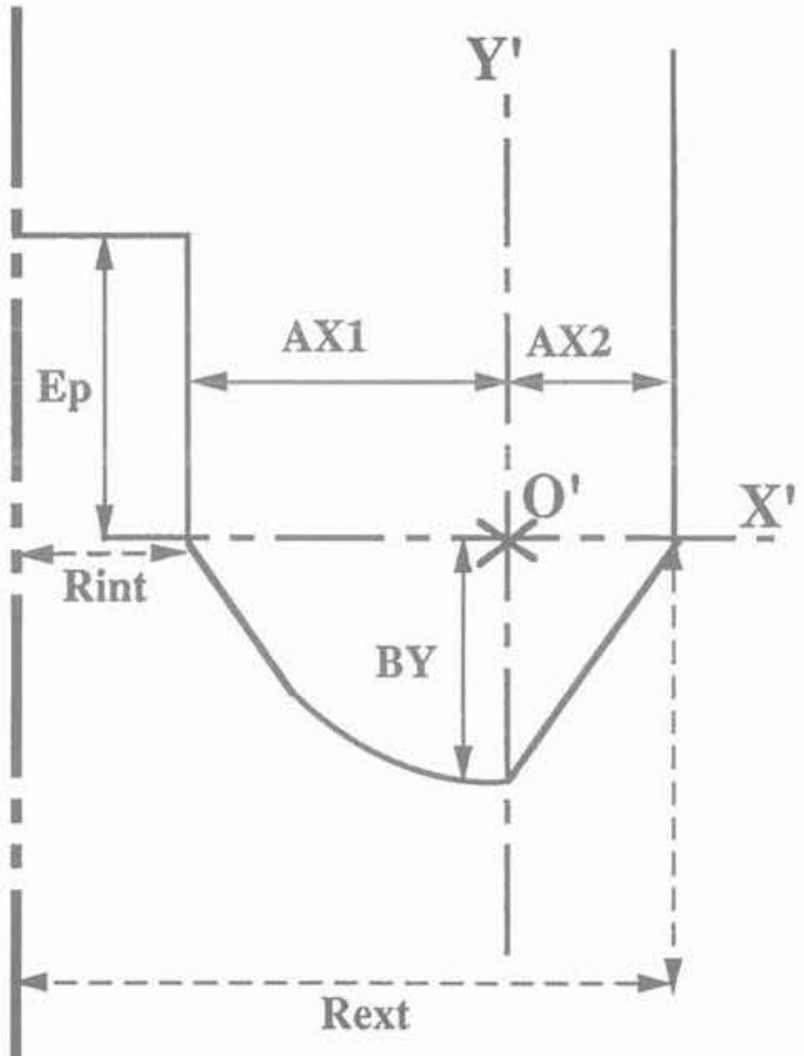

Fig. 10. - Paramètres définissant la forme du fond de la saignée.

Fig. 10. - Parameters of the type and shape of the core bit.

Tableau 2. - Récapitulation des principaux résultats sur l'influence de la forme de la saignée sur l'amorce de la rupture.

Table 2. - Summing up of the principal results about the influence of core bit shape on rupture initiation.

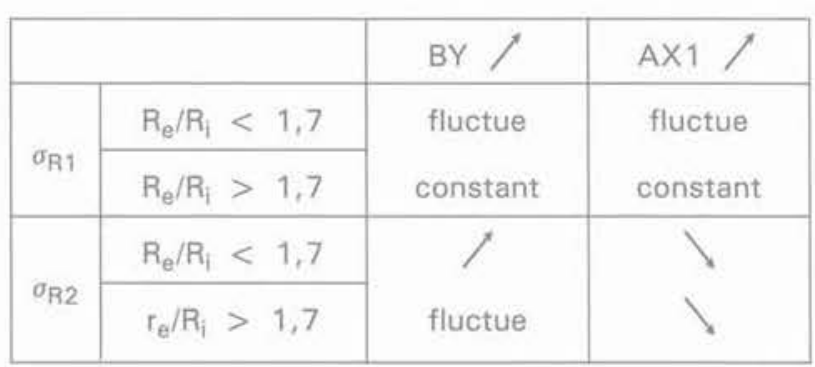

pétroliers en permettant de sélectionner les données de forage afin d'obtenir des carottes saines. L'étude de la variation du domaine de non discage ou plus exactement du domaine de sécurité vis-à-vis de l'amorce de la rupture va dans ce sens.

Nous avons souligné :

- qu'il existe des relations linéaires liant $\sigma_{\mathrm{R} 1}$ et $\sigma_{\mathrm{R} 2}$ à la résistance à la compression et au cisaillement du matériau et à la densité de boue ;

- que le paramètre rapport des rayons du carottier est important et qu'il est associé à une épaisseur de 


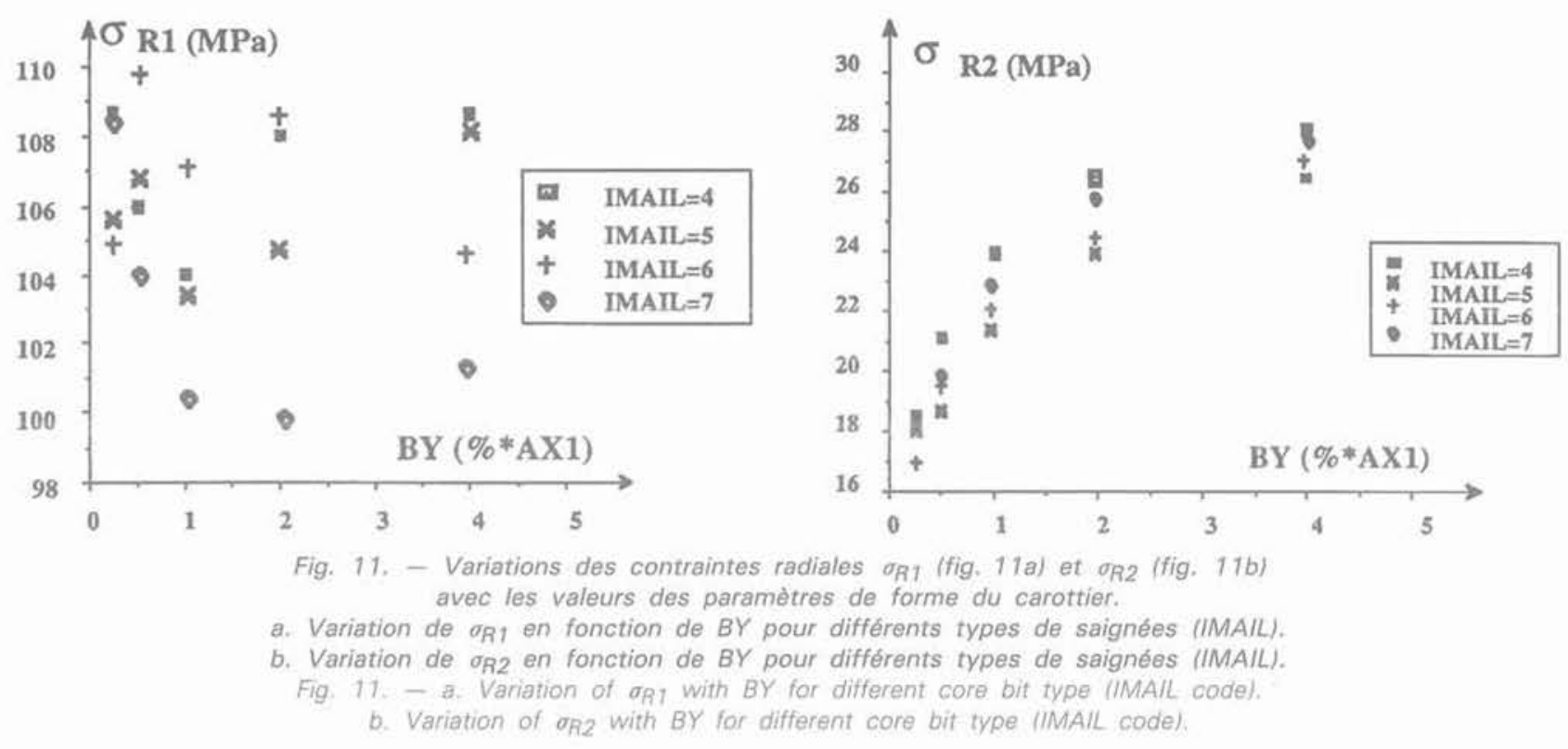

disque potentiel ainsi qu'à un mode de rupture (trois classes de disques ont été répertoriées selon leurs épaisseurs) ;

- que la forme et la géométrie du carottier influent sur les contraintes d'initialisation de la rupture. Le sens de variation de $\sigma_{\mathrm{R} 1}$ et $\sigma_{\mathrm{R} 2}$ en fonction de ces paramètres est soumis aux valeurs de la contrainte axiale, des caractéristiques géomécaniques du matériau et la densité de boue.

Les perspectives envisagées à court terme sont, d'une part la transcription du logiciel en mode tridimensionnel, et d'autre part, l'introduction dans DISCAGELF d'une panoplie complète des différents carottiers existants (avec leurs exigences) ainsi que les problèmes d'écoulement de fluide.

\section{REMERCIEMENTS}

Les auteurs remercient la Société Nationale Elf Aquitaine Production, et particulièrement MM. MAURY et SANTARELLI pour leur soutien, leur disponibilité et leurs précieux conseils. L'étude conduisant au doctorat de V. VENET a été financée par Elf Aquitaine.

\section{BIBLIOGRAPHIE}

(1) BOUMADHI M. (1990), Conception et réalisation d'un triaxial vrai de grande capacité simu. lateur d'ouvrages souterrains. Thèse de Doctorat à l'Universite de Lille I.

(2) CHEATHAM J.M. (1984), Wellbore stability. J. of Petr. Tech., June pp. 889-896.

(3) DURELLI A.J., OBERT L., PARKS V.J. (1968), Stress required to initiale core disking. Society of Mining Engineers Trans., Vol. 241, pp. $269-276$.

(4) DYKE C.G. (1989), Core disking. Its potential as an indicator of principal in situ stress directions. Symposium rock at great depth Pau, Vol.
3, pp., 1057-1065, Maury, Fourmaintraux rédacteurs.

(5) GUÉNOT A. (1989), Borehole breakouts and stress fields. Int. J. Rock Mech. Min. Sci. Geomech. Abstract, Vol. 26, n 314 , pp. 185-195.

(6) ISAEV V. (1977), Influence du facteur d'échelle sur le discage des carottes provenant de sondages de différents diamètres (en russe). Trudy VNIMI (Leningrad). Recueil 103, pp. 112-116.

(7) MIGUEZ R. (1990), Etude du discage. Application à la détermination des contraintes in situ lorsque le forage n'est pas chargé. Thèse Doctorat en mécanique des solides, Université de Lille 1.

(8) MOUDAFI M. (1986), Etude expérimentale de la réactivité des roches par foration sous contraintes. Thèse de Doctorat de l'Institut National Polytechnique de Lorraine (Nancy).

(9) OBERT L., STEPHENSON D.E. (1965), Stress conditions under which core discing occurs. Society of Mining Engineers Trans., Vol. 232, pp. 227-235.

(10) PAILLET F.L., KIM K. (1987), Character and distribution of borehole breakouts and their relationship to in situ stresses in deep Columbia River Basalts. J. of Geophysical Research, Vol. 92, n’ 137, pp. 6223-6224.

(11) PANET M. (1969), Quelques problèmes de mécanique des roches posés par le tunnel du Mont-Blanc. Bulletin de Liaison Labo. Routiers Ponts et Chaussées, n 42, pp. 115-145.

(12) STACEY T.R. (1982), Contribution to the mechanism of core discing. Journal of the South African, Institute of Mining and Metallurgy, pp. 269-274.

(13) SUGAWARA K., KAMEOKA Y., SAITO T., GKA Y., HIRAMATSU Y. (1978), Etudes sur 
le phénomène de discage des carottes. Journal Min. Inst. Japan, Vol. 94, pp. 797-803.

(14) VENET V., HENRY J.P., SANTARELLI F., MAURY V. (1989), Modélisation du discage pour l'estimation des contraintes à grande profondeur. Symposium Rock at great depth Pau, Vol. 3, pp. 1551-1557, Maury, Fourmaintraux rédacteurs (août 1989).
(15) VENET V. (1991), Modélisation de l'initialisation du discage par éléments finis : élaboration d'une méthode d'optimisation de carottage. Thèse de Doctorat de l'Université des Sciences et Techniques de Lille, $163 \mathrm{pp}$.

(16) ZHU W., LI G., WANG K. (1985), Analysis of disking phenomenon and stress field in the region of an undergroun powerhouse. Rocks Mechanic and Rock Engineering, $n^{\circ} 18, p p .1-15$. 